\title{
Measuring Program Comprehension with Functional Magnetic Resonance Imaging
}

\author{
Janet Siegmund, Sven Apel, Christian Kästner, Chris Parnin, \\ Anja Bethmann, Gunter Saake, Thomas Leich, and André Brechmann
}

\begin{abstract}
Programmers spend significant time comprehending source code everyday, so naturally, decades of research have been devoted to understanding and improving program comprehension. However, this research has still not completely unraveled what happens inside programmersâ heads when they work with source code. The problem is that program comprehension is an internal cognitive process that inherently eludes measurement. To shed light on this issue, we explored the feasibility of using functional magnetic resonance imaging (fMRI), which has proved successful in cognitive neuroscience to understand cognitive processes in more depth.
\end{abstract}

fMRI measures and locates change of the oxygen level in the blood, which is an indicator for activation. To filter irrelevant activation that is not specific to program comprehension (e.g., visual perception of source code), fMRI measurement requires a control condition that does not require program comprehension. Then, the difference in activation between program comprehension and the control condition describes the brain areas that are specific for program comprehension.

We performed our study as outlined in Figure 1 and described it in more detail in the corresponding publication $\left[\mathrm{SKA}^{+}{ }^{14}\right]$. We designed twelve source-code snippets of similar length and difficulty (e.g., reversing a word, computing the cross sum of a given number; see project's website at $t$ inyurl . com/ProgramComprehensionAndfMRI / for all snippets), which we evaluated in pilot studies $\left[\mathrm{SBA}^{+} 12\right]$. We asked participants to determine the output of each source-code snippet. We obfuscated identifier names, so that they do not give a hint about a snippet's purpose. As control condition, we introduced syntax errors into the twelve code snippets and asked participants to locate them. As participants, we recruited computer-science students with a similar level of programming experience (as determined by our questionnaire $\left[\mathrm{SKL}^{+} 14\right]$ ). A trial consisted of a comprehension task and a syntax task (different snippets), each followed by a resting condition to let the oxygen level return to a baseline. Overall, the experiment consisted of twelve trials.

As a result of our experiment, we observed a clear activation pattern of five different brain areas located in the left hemisphere(see Figure 1(c)), which are associated with different cognitive processes: language comprehension, working memory, problem solving, and divided attention. Thus, we can hypothesize that the following happened during program comprehension: Participants processed the words and symbols of the code snippets (language processing) and kept variable values in mind (working memory). At the same time (divided attention), they analyzed the effect of the words and symbols on the variable values (problem solving). Hence, the activated brain areas and associated processes align 


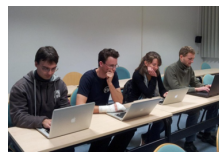

(a) Prestudy

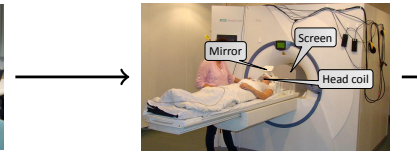

(b) fMRI measurement

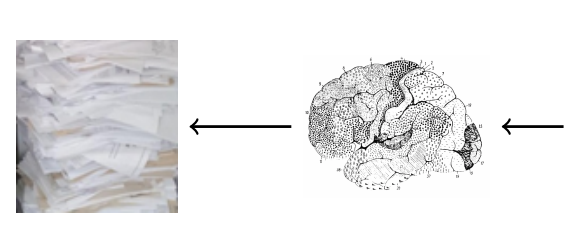

(e) Other fMRI studies (d) Cognitive processes

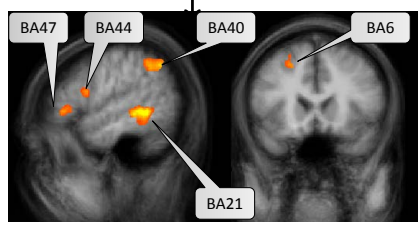

(c) Activation pattern

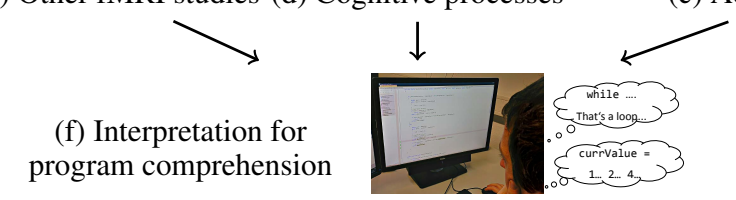

Figure 1: Workflow of our fMRI study.

well with our current understanding of program comprehension, backing up decades of program-comprehension research with first supporting evidence from neuro-imaging data. The finding that all activated areas were located in the left hemisphere, however, suggests that processes related to language competences are most relevant.

Furthermore, our experiment demonstrates the feasibility of measuring program comprehension with fMRI, and we hope that other researchers will follow us to eventually unravel the mysteries of programmers' brains and to enrich the empirical basis for future software-engineering research, tools, and education. For more details on this work, we refer the reader to the paper "Understanding Understanding Source Code with Functional Magnetic Resonance Imaging", which was published at the International Conference on Software Engineering in 2014 [SKA $\left.{ }^{+} 14\right]$.

\section{References}

$\left[\mathrm{SBA}^{+}\right.$12] Janet Siegmund, André Brechmann, Sven Apel, Christian Kästner, Jörg Liebig, Thomas Leich, and Gunter Saake. Toward Measuring Program Comprehension with Functional Magnetic Resonance Imaging. In Proc. Int'l Symposium Foundations of Software Engineering-New Ideas Track (FSE-NIER), pages 24:1-24:4. ACM, 2012.

[SKA $\left.{ }^{+} 14\right]$ Janet Siegmund, Christian Kästner, Sven Apel, Chris Parnin, Anja Bethmann, Thomas Leich, Gunter Saake, and André Brechmann. Understanding Understanding Source Code with Functional Magnetic Resonance Imaging. In Proc. Int'l Conf. Software Engineering (ICSE), pages 378-389, 2014.

$\left[\mathrm{SKL}^{+}{ }^{14}\right]$ Janet Siegmund, Christian Kästner, Jörg Liebig, Sven Apel, and Stefan Hanenberg. Measuring and Modeling Programming Experience. Empirical Softw. Eng., 19(5), 2014. Invited article. 